\title{
Extended High Circular Polarization in the Orion Massive Star Forming Region: Implications for the Origin of Homochirality in the Solar System
}

\author{
Tsubasa Fukue • Motohide Tamura • Ryo Kandori • Nobuhiko Kusakabe • \\ James H. Hough • Jeremy Bailey • Douglas C. B. Whittet • Philip W. Lucas • \\ Yasushi Nakajima • Jun Hashimoto
}

Received: 18 December 2009 / Accepted: 13 January 2010/

Published online: 7 March 2010

(C) The Author(s) 2010. This article is published with open access at Springerlink.com

\begin{abstract}
We present a wide-field $\left(\sim 6^{\prime} \times 6^{\prime}\right)$ and deep near-infrared $\left(K_{s}\right.$ band: $\left.2.14 \mu \mathrm{m}\right)$ circular polarization image in the Orion nebula, where massive stars and many low-mass stars are forming. Our results reveal that a high circular polarization region is spatially extended $(\sim 0.4 \mathrm{pc})$ around the massive star-forming region, the BN/KL nebula. However, other regions, including the linearly polarized Orion bar, show no significant circular polarization. Most of the low-mass young stars do not show detectable extended structure in either linear or circular polarization, in contrast to the BN/KL nebula. If our solar system formed in a massive star-forming region and was irradiated by net circularly polarized radiation, then enantiomeric excesses could have been induced, through asymmetric photochemistry, in the parent bodies of the meteorites and subsequently delivered to Earth. These could then have played a role in the development of biological homochirality on Earth.
\end{abstract}

T. Fukue $(\bowtie) \cdot$ M. Tamura $\cdot$ R. Kandori $\cdot$ N. Kusakabe $\cdot$ Y. Nakajima

National Astronomical Observatory of Japan, 2-21-1 Osawa, Mitaka, Tokyo 181-8588, Japan e-mail: tsubasa.fukue@nao.ac.jp

M. Tamura $\cdot$ J. Hashimoto

Graduate University of Advanced Science, 2-21-1 Osawa, Mitaka, Tokyo 181-8588, Japan

J. H. Hough • P. W. Lucas

Centre for Astrophysics Research, Science and Technology Research Institute,

University of Hertfordshire, Hatfield, Herts AL10 9AB, UK

J. Bailey

School of Physics, University of New South Wales, NSW 2052, Australia

D. C. B. Whittet

New York Center for Astrobiology and Department of Physics and Astronomy,

Rensselaer Polytechnic Institute, Troy, NY 12180-3590, USA

Y. Nakajima

Department of Astrophysics, Nagoya University, Nagoya 464-8602, Japan 
Keywords Circular polarization $\cdot$ Orion nebula $\cdot$ Enantiomers $\cdot$ Homochirality $\cdot$

Origins of life

\section{Introduction}

The origin of biomolecular homochirality, which refers to the phenomenon that terrestrial living material consists almost exclusively of one enantiomer, left-handed amino acids and right-handed sugars, is a longstanding mystery that is critical to understanding the origin and development of life (Bonner 1991, 1995; Meierhenrich and Thiemann 2004; Barron 2008). Amino acids in several meteorites (e.g., Murchison, Murray, Orgueil) have been found to have enantiomeric excesses (EEs) of the same handedness as that seen in biological amino acids (Cronin and Pizzarello 1997; Pizzarello and Cronin 2000; Pizzarello et al. 2003; Pizzarello et al. 2008; Glavin and Dworkin 2009; Sephton 2002). Such detection of EEs in meteorites is consistent with the hypothesis that life on Earth was seeded by the delivery of organics from outer space during the heavy bombardment phase of Earth's early history (Bailey et al. 1998; Bailey 2001; Buschermöhle et al. 2005). Furthermore, homogeneity of right-handed sugars may be also be initiated by exogenous injection of low EEs of amino acids as a catalyst (Weber 2001; Pizzarello and Weber 2004; Córdova et al. 2005; Córdova et al. 2006).

Amino acids or amino acid precursors (Botta and Bada 2002) can exist in space conditions. Amino acids were obtained in laboratory experiments that simulate ultraviolet (UV) photolysis of interstellar ice analogues (Bernstein et al. 2002; Muñoz-Caro et al. 2002; Nuevo et al. 2008). Experiments have indicated that cosmic rays can produce amino acid precursors in icy environments (Hudson et al. 2008). However, external effects seem to be necessary to produce EEs (Bonner 1991, 1995).

EEs can be produced by circularly polarized light (CPL) through asymmetric photochemistry, such as asymmetric photolysis or synthesis (Griesbeck and Meierhenrich 2002; Meierhenrich and Thiemann 2004; Meierhenrich et al. 2005a) as shown in laboratory experiments. Significant EEs $(\sim 20 \%)$ have been reported in the products of asymmetric photolysis from a racemate (Bonner 1991, 1995). The handedness of EEs via asymmetric photolysis depends on the sign of the CPL due to the difference in photolysis rate of L- and D-enantiomers (Bonner 1991). Even elliptical polarization can induce asymmetric photolysis (Bonner and Bean 2000). The amino acid leucine in the solid state has been photolysed in the laboratory (Meierhenrich et al. 2005b). Furthermore, by irradiation of CPL on interstellar ice analogues, small EEs of less than about $1 \%$ have been obtained in laboratory experiments (Nuevo et al. 2006). The possibility of asymmetric synthesis of amino acid precursors in interstellar complex organics using CPL has been demonstrated in a recent experiment by Takano et al. (2007). They prepared complex organic compounds from proton-irradiated gas mixtures as interstellar analogues, and reported EEs of $+0.44 \%$ by right-circularly polarized UV light and of $-0.65 \%$ by left-circularly polarized UV light. Amplification of initially low EEs, through autocatalysed reactions, have been experimentally demonstrated (Soai et al. 1995; Shibata et al. 1998; Soai and Kawasaki 2006). Other recent experiments have shown that asymmetric amplification under solid-liquid equilibrium conditions of serine with $1 \% \mathrm{EE}$ can produce EEs of greater than 99\% (Klussmann et al. 2006).

Astronomical sources of CPL that might induce EEs in interstellar material have been investigated. Neutron stars were originally suggested as a possible source of CPL (Rubenstein et al. 1983; Bonner 1991). However neutron stars are not significant sources of CPL at visible and UV wavelengths (Bailey 2001). Bailey et al. (1998) proposed that CPL produced in star-forming regions could contribute to producing the astronomical EEs 
through asymmetric photolysis. Previous observations indicate that regions of massive starformation have higher degrees of CPL, although only a relatively small number of starforming region have been observed (Clayton et al. 2005).

The origin of life and homochirality may be closely related to the formation process for solar-mass stars and their planetary systems. Low mass stars such as the Sun can be formed in massive star-forming regions such as the Orion nebula or relatively isolated regions where only low-mass stars are formed, such as Taurus (Hester and Desch 2005). However, isotopic studies of meteorites that confirm the presence of short half-life radionuclides such as ${ }^{60} \mathrm{Fe}$ (with a half-life of $1.5 \mathrm{Myr}$ ) in the young solar system suggest that a supernova explosion occurred near the Sun (Hester et al. 2004, Hester and Desch 2005, Mostefaoui et al. 2005, Tachibana et al. 2006), indicating the birth of the solar system in a massive star-forming region.

The Orion nebula is the nearest star-forming region in which both high-mass and low-mass stars are being formed (Hillenbrand 1997), and it serves as a valuable test-bed for investigating the CPL mechanism for the origin of EEs. The entire Orion nebula consists of a variety of star forming processes (Genzel and Stutzki 1989; O’Dell 2001). At the core of the Orion nebula, there is a group of massive (OB-type) young stars, the Trapezium cluster (see also Fig. 1). Around the Trapezium, the Orion nebula harbors the association of many young stars with various mass ranges, the Orion Nebula Cluster (ONC). The embedded massive star-forming region, the $\mathrm{BN} / \mathrm{KL}$ nebula, is located near the Trapezium. The $\mathrm{BN} / \mathrm{KL}$ nebula harbors massive protostellar objects such as the BN object and IRc2, with masses of $>7$ and 25 solar masses, respectively (Genzel and Stutzki 1989). Several young massive stars such as Source I and SMA1 are also thought to exist very close to IRc2 (Gezari 1992; Beuther et al. 2004). The BN object seems to be in an earlier phase of star formation than the Trapezium (Jiang et al. 2005), as well as the deeply embedded sources such as IRc2. The Trapezium stars appear to have evacuated a cavity, near the surface of the molecular cloud OMC-1 (Genzel and Stutzki 1989; O'Dell 2001). The evacuation of the near-side of the cloud by the Trapezium provides lower extinction to aid observations. Furthermore, background stellar contamination in the Orion nebula is negligible due to the dense molecular cloud behind, and foreground contamination is also relatively low (Jones and Walker 1988; Getman et al. 2005).

As many of the low-mass YSOs will evolve into Sun-like stars, studies of the Orion starforming region enable us to investigate processes that may have occurred during the birth of our own solar system. In particular, we can explore the circularly polarized radiation that may have bathed the nascent solar system. The obscuring dust prevalent in star-forming regions can be penetrated with observations at near-infrared (NIR) wavelengths which can, thus, be used to study the scattering processes in the circumstellar structures of young stars.

NIR linear polarization (LP) images of the Orion nebula have been reported on a range of scales (e.g., Minchin et al. 1991; Jiang et al. 2005; Simpson et al. 2006). The NIR three color linear polarimetry by Tamura et al. (2006) revealed the extensive $(>0.7 \mathrm{pc})$ LP nebulae around IRc2 and BN. In addition, they reported several small linearly polarized nebulae, the linearly polarized Orion bar, and the low LP near the Trapezium. The LP of hundreds of ONC stars in this region was also investigated, showing the typical hourglassshaped magnetic field pattern (Kusakabe et al. 2008).

NIR circular polarization (CP) images of the Orion nebula have been reported that reveal a quadrupolar distribution of right- and left-handed CP in the vicinity of OMC-1 (Bailey et al. 1998; Chrysostomou et al. 2000). CP imaging of the Orion BN/KL region show that the quadrupolar structure is centered around the young star IRc2, which appears to be dominant for the large CP (Buschermohle et al. 2005; Fukue et al. 2009). The spatial extent of high CP emission and the degree to which highly polarized radiation interacts with other young stars can only be investigated by extending the spatial coverage of the observations. 
A first such attempt was reported by Buschermohle et al. (2005), who found generally low degrees of CPL toward several segements of the adjacent HII region. In this paper, we report a deep, wide-field $\left(\sim 6^{\prime} \times 6^{\prime}\right)$ NIR CP image in the $K_{s}$ band $(2.14 \mathrm{um})$ of the Orion nebula. Moreover, aperture polarimetry for several hundred point-like sources is also reported. Based on polarimetry results, we discuss possible implications for the origin of EEs, with a view to testing this mechanism for the origin of biological homochirality.

\section{Observations and Data Reduction}

$2.14 \mu \mathrm{m}\left(K_{s}\right.$ band) and $1.63 \mu \mathrm{m}(H$ band) imaging circular polarimetry data of M42 were obtained with the SIRIUS camera (Nagayama et al. 2003) and its polarimeter on the 1.4-m IRSF telescope at the South African Astronomical Observatory, on nights during 2006 December. These observations and subsequent data reduction were the same as described in Fukue et al. 2009 (the resultant stellar seeing size $\sim 1.5^{\prime \prime}$ ), although their observations focus just on the $\mathrm{BN} / \mathrm{KL}$ region. The estimated uncertainties in the degrees of $\mathrm{CPL}$ range from $\sim 0.3 \%$ to $\sim 1 \%$ close to the corners of the CP image.

$2.14 \mu \mathrm{m}\left(K_{s}\right.$ band) imaging linear polarimetry of M42 was obtained with the SIRIUS camera and its polarimeter on the IRSF telescope, on the night of 2005 December 26, with seeing similar to that in the circular polarization observations. These observations and subsequent data reduction were the same as described in Tamura et al. 2006 (see also Kandori et al. 2006; Tamura et al. 2003), with estimated uncertainties less than about $0.3 \%$.

Software aperture circular polarimetry for 540 point-like sources, with intensity signalto-noise $>10$, was carried out in a manner similar to that used for linear polarimetry in Kusakabe et al. (2008), and using the same aperture radius of 3 pixels. A total of 353 sources had a polarization signal-to-noise ratio $>10$ in both the $H$ and $K_{\mathrm{s}}$ bands.

\section{Results and Discussion of Polarimetry}

Figure 1 shows the wide-field images of circular and linear polarization of the Orion starforming region in the $K_{s}$ band $(2.14 \mu \mathrm{m})$. The field-of-view is 5.5 arcminutes square. The Trapezium is indicated around the center in Fig. 1. The north-west area with strong CP corresponds to the embedded massive star-forming region, the BN/KL nebula, containing the massive protostars IRc2 and BN. These massive protostars are also located near the center of the large LP region in Fig. 1b (Tamura et al. 2006). Point-like sources are not completely cancelled and are visible in the image even if they are unpolarized, because the seeing size changes during the observations of images taken at different quarter-waveplate angles. Since our frame registration is not performed in a sub-pixel unit, the residual stellar profiles on the Stokes $V$ image can be seen as a close pair of positive and negative peaks. This does not affect the polarimetry of extended nebulae on the Stokes $V$ image or the aperture polarimetry of point-like sources performed using each waveplate angle image. The faint circular patterns centered on, and to the south of, the Trapezium in the CP image are ghost images caused by the polarimeter optics.

Our wide-field image in Fig. 1 reveals that the $\mathrm{CP}$ region around the $\mathrm{BN} / \mathrm{KL}$ nebula extends over a large region (up to $\sim 0.4 \mathrm{pc}$ ). The degrees of $\mathrm{CP}$ are very large, ranging from $+17 \%$ to $-5 \%$, which is consistent with previous polarimetry measurements (Bailey et al. 1998; Chrysostomou et al. 2000; Buschermöhle et al. 2005). The CP map reported in 

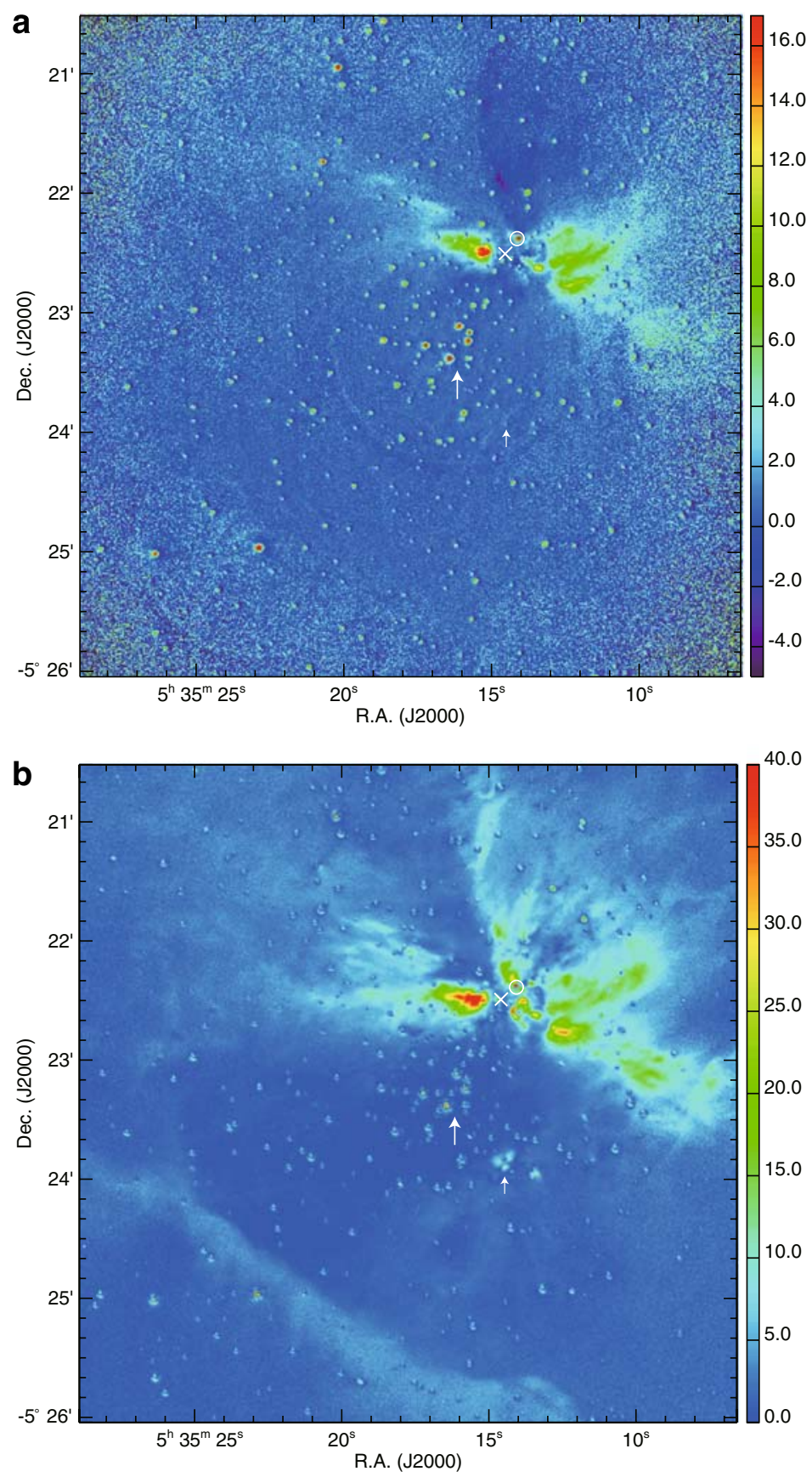

Fig. 1 Image of degree of polarization $(\%)$ in the $K_{s}$ band $(2.14 \mu \mathrm{m})$ of the central region of the Orion starforming region. a Image of circular polarization degree; $\mathbf{b}$ The degree of linear polarization. The field-of-view is 5.5 arcminutes or $0.74 \mathrm{pc}$ square at a distance of $460 \mathrm{pc}$. North is up and east is to the left. The positions of IRc2 and $\mathrm{BN}$ are indicated by a cross and a circle, respectively, while those of the Trapezium stars and the low-mass young star OMC-1S are denoted by big and small arrows, respectively. A positive sign for CP indicates that the electric vector is rotated anticlockwise in a fixed plane relative to the observer 

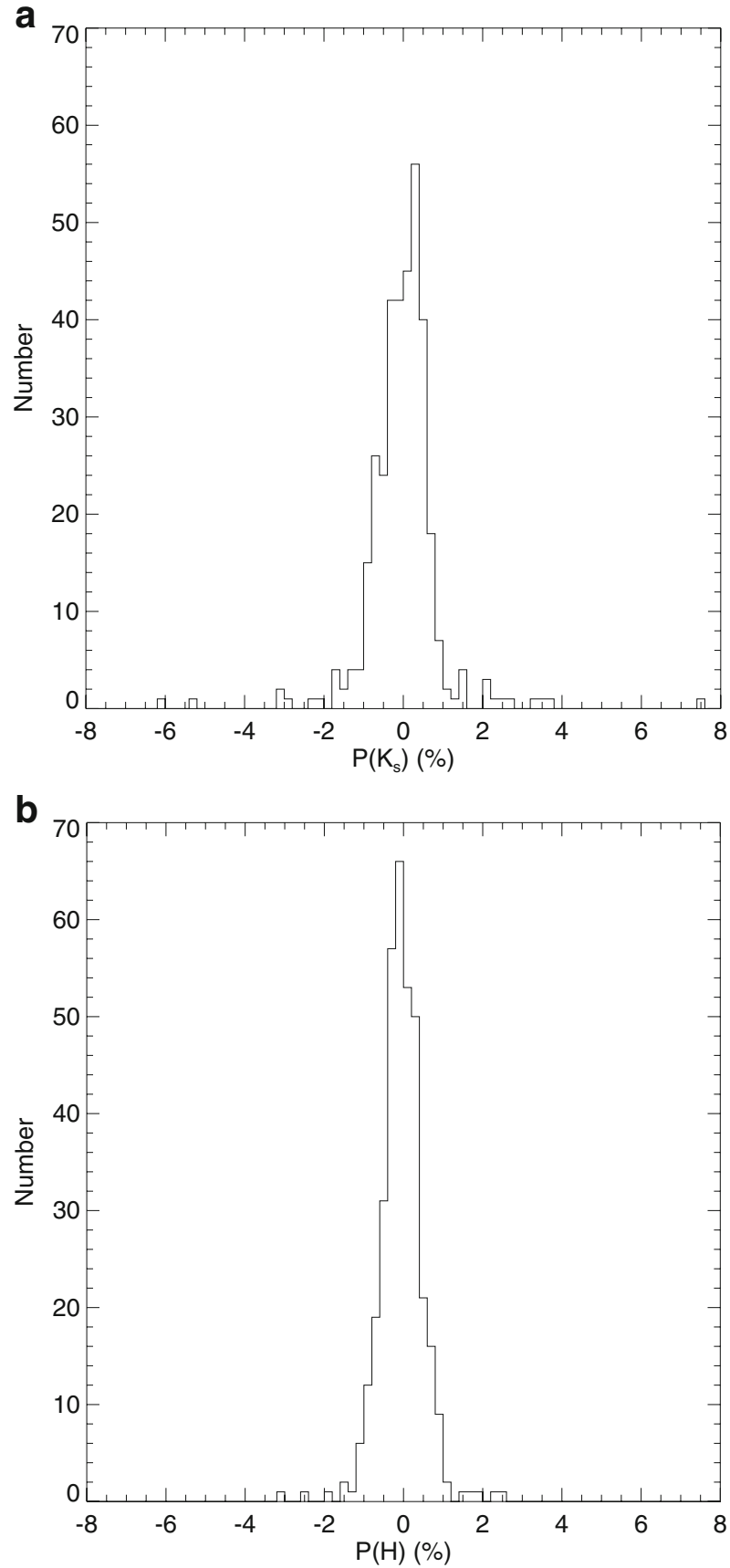

Fig. 2 Histograms of circular polarization degree (\%) of 353 point-like sources. a in the $K_{s}$ band $(2.14 \mu \mathrm{m})$; $\mathbf{b}$ in the $H$ band $(1.63 \mu \mathrm{m})$. The histograms are constructed using a bin width of $0.2 \%$ 


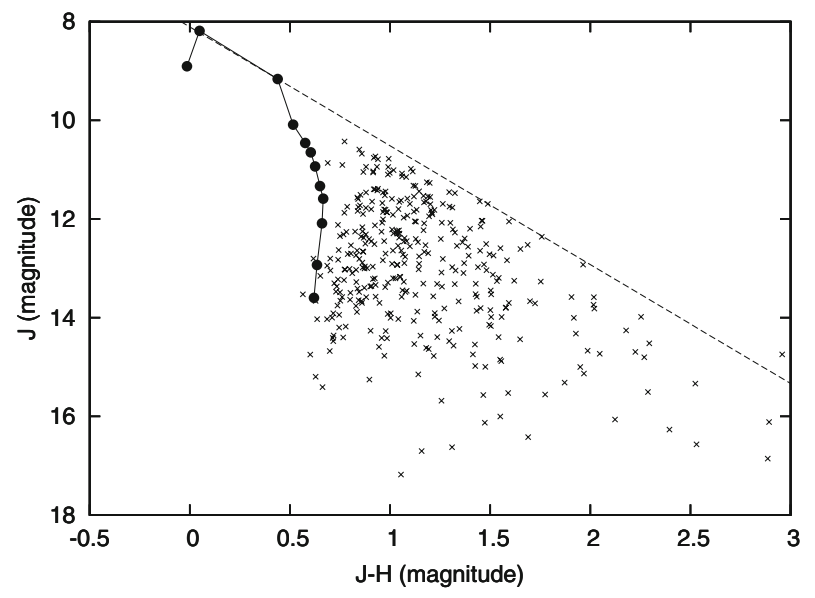

Fig. 3 Color-magnitude diagram for 353 point-like sources used in Fig. 2, using their $J$-band (1.25 $\mu \mathrm{m})$ and $H$-band $(1.63 \mu \mathrm{m})$ data in the same observation. The vertical axis shows $J$ magnitude, and the horizontal axis shows $J-H$ magnitude. Our observational data are plotted with crosses. The filled circles denote the loci of 1 Myr old PMS stars at $460 \mathrm{pc}$, according to the stellar evolution model by Testi et al. (1998). The assumed masses are $0.1,0.2,0.4,0.6,0.8,1,1.2,1.5,2,2.5,3$, and 3.5 solar masses, from bottom to top (the second point from the top for 3.5 solar masses), connected by the solid line. The dashed line identifies the reddening law through the loci of the 2.5 solar masses (Cohen et al. 1981)

this study covers a much larger area than in previous studies. It reveals that significant CP extends over a region $\sim 400$ times the size of the solar system (assumed to be $200 \mathrm{AU}$ in diameter, including trans-neptunian objects). This extension of the $\mathrm{CP}$ region is almost comparable to the size of the linearly polarized region in Fig. 1b (Tamura et al. 2006).

There exists no significant CP around the Trapezium, in contrast with the BN/KL region. In particular, the linearly polarized Orion bar in Fig. 1b (Tamura et al. 2006) shows no significant CP in Fig. 1a. The centrosymmetric LP vector pattern indicates that the polarized Orion bar is irradiated by the Trapezium stars (Tamura et al. 2006). This indicates that the first scattering of the incident radiation from the Trapezium stars by the grains within the bar cannot produce the significant $\mathrm{CP}$; this in turn shows that the dust grains in the LP bar are not well aligned (Gledhill and McCall 2000). The colors of this region show that the Trapezium and the bar are located near the surface of the cloud (Buschermöhle et al. 2005 ) in contrast with the BN/KL region.

Most of the low- or medium-mass young stars in Fig. 1 do not show extended structure in either LP or CP, in contrast to the BN/KL region. Even those with a NIR nebula that is linearly polarized (e.g., OMC-1S, see Tamura et al. 2006; see also Fig. 1), show no significant $\mathrm{CP}$, even when the nebula is spatially resolved. Figure 2 shows the distribution of the aperture circular polarimetry, for the 353 point-like sources detected both in the $K_{s}$ band and $H$ band with a polarization signal-to-noise ratio $>10$. Many of these sources are low-mass young stars whose circumstellar structures are unresolved at a 1.5 -arcsecond resolution (equivalent to about $700 \mathrm{AU}$ ). Figure 3 shows a $J-H$ vs. $J$ color-magnitude diagram for these sources. In the diagram, we indicate the locus of $1 \mathrm{Myr}$ old pre-main sequence (PMS) stars at the distance of $460 \mathrm{pc}$ based on the stellar evolution model by Testi et al. (1998). Also we indicate the reddening direction based on Cohen et al. (1981). The diagram is consistent in indicating that these sources are 1-Myr old PMS stars with masses less than $\sim 3$ solar masses. The vast majority of these sources measured in this study are 
cluster members (Jones and Walker 1988; Getman et al. 2005; Hillenbrand 1997; Lucas et al. 2001). The proper motions and radial velocities of ONC members show a dispersion of a few $\mathrm{km} \mathrm{s}^{-1}$ (Jones and Walker 1988; Fürész et al. 2008), implying that these stars will move within about 1 pc, in 1 Myr. In Fig. 2, the measured degree of CP for each source is generally small. We conclude that none of the detected point sources clearly show significant integrated circular polarizations (>than $1.5 \%$ both in $K_{s}$ and $H$ bands in the same handedness); one source does have a CP $>1.5 \%$, both in the $K_{s}$ and $H$ bands, but is embedded in the western high $\mathrm{CP}$ region and hence substantially contaminated. OMC-1S shows aperture circular polarimetry of about $0.3 \%$ in $K_{S}$ band. These results are consistent with previous observations (Clayton et al. 2005).

\section{$\mathrm{CP}$ in Massive Star-forming Regions: Possible Implications for the Origins of Homochirality}

We will now discuss the implications of these results for the origin of biomolecular homochirality. Bailey (2001) discusses how CPL in star-forming regions might be important in producing EEs and ultimately seeding homochirality on terrestrial planets. Imaging circular polarimetry of several YSOs (Gledhill et al. 1996; Chrysostomou et al. 1997; Bailey et al. 1998; Chrysostomou et al. 2000; Clark et al. 2000; Ménard et al. 2000; Chrysostomou et al. 2007; Fukue et al. 2009; Clayton et al. 2005) and numerical simulations (Fischer et al. 1996; Wolf et al. 2002; Whitney and Wolff 2002; Lucas et al. 2004; Lucas et al. 2005; Chrysostomou et al. 2007) indicate that a YSO will not usually generate a net $\mathrm{CP}$ because it will have regions of positive and negative sign that cancel globally. Hence, a nascent solar system around a low-mass star would not be irradiated by a net CP. A low-mass YSO would only experience strong CP of a single sign when it is externally irradiated by a high-mass YSO. In our polarimetry results, low-mass young stars themselves do not show strong one-handed CP. On the other hand, extended regions of high $\mathrm{CP}$ (hundreds of times the size of the solar system) are associated with high-mass stars. Large numbers of low-mass YSOs are often located in a clustered star-forming region containing massive stars. The high stellar density $\left(>10^{3}\right.$ stars $\left.^{\mathrm{pc}^{-3}}\right)$ and the large and wide $\mathrm{CP}$ region around the location of IRc2 suggest that there are at least several stars in the high $\mathrm{CP}$ region around IRc2. There, a low-mass young star can see predominantly onehandedness of $\mathrm{CP}$, which provides an external source for asymmetric photolysis to yield EEs in any chiral molecules (Bailey 2001; Bonner 1991).

Photolysis of amino acids requires UV radiation, rather than the infrared radiation observed in this study. UV radiation cannot be directly observed as it is unable to penetrate the dust that lies along the line-of-sight between the Earth and regions of high CP. Numerical calculations (Bailey et al. 1998) indicate that significant amounts of UV CP can be produced by young stars and this could spread over large distances because of the large cavities formed by bipolar outflows and jets (Tamura et al. 2006). UV CP can then be produced by mechanisms discussed by Lucas et al. (2005).

Should the asymmetric photochemical processes reported in laboratory experiments operate in regions of high-mass star-formation, then they could give rise to the observed EEs of meteoritic amino acids, possibly amplified through autocatalysis. Assuming that the observed EEs were produced in the nascent solar system, the detection of EEs of meteoritic amino acids on Earth suggests that the EEs can survive for many billions of years. Our observation of wide regions of high $\mathrm{CP}$ suggests that similar CP could have irradiated the early solar system if it formed in a similar environment. Recently, Glavin and Dworkin 
(2009) have detected no L-isovaline excess for the most pristine Antarctic CR2 meteorites Elephant Moraine 92042 and Queen Alexandra Range 99177, whereas they have detected large L-EEs in the CM meteorite Murchison and the CI meteorite Orgueil. They discuss the possibility that the detected EEs may be produced by amplification of small initial EEs during an aqueous alteration phase.

The high spatial extent of large degrees of CPL, together with the various laboratory experiments, supports the idea that the initial seeds of homochirality are generated in the nascent solar system and are carried to Earth during the heavy bombardment that occurred in the Earth's early history (Bailey et al. 1998), with subsequent chiral amplification (Barron 2008; Soai and Kawasaki 2006; Klussmann et al. 2006).

The above scenario for the origin of homochirality requires that the Sun be formed near a cluster of massive stars (Hester and Desch 2005), which produce most of the scattered light over a very large region of a few pc. Stars can also form in relative isolation in a molecular cloud that forms only low-mass stars. That the solar system originated in a massive star formation region is supported by isotopic studies of meteorites such as ${ }^{60} \mathrm{Fe}$ suggesting that a supernova explosion occurred near the Sun (Mostefaoui et al. 2005; Tachibana et al. 2006). The possibility that the solar protoplanetary disk survived even a supernova explosion is supported by numerical simulations (Ouellette et al. 2007).

\section{Conclusion}

CPL, produced in regions of high-mass star-formation, is one possibility for producing EEs in small bodies in the presolar nebula, which could then be delivered to the early Earth, thereby contributing to the evolution of homochirality in living organisms. NIR wide-field $\left(\sim 6^{\prime} \times 6^{\prime}\right)$ imaging circular polarimetry of the core of the Orion nebula show that high CP extends to $\sim 0.4 \mathrm{pc}$ around the massive star-forming region, the $\mathrm{BN} / \mathrm{KL}$ nebula. This extension of CP is comparable with that of LP. On the other hand, the area other than the massive star forming region generally showed low $\mathrm{CP}$, and most of the low- or mediummass young stars do not show detectable extended structure associated with them in either $\mathrm{LP}$ or $\mathrm{CP}$, in contrast to the $\mathrm{BN} / \mathrm{KL}$ region. Even $\mathrm{OMC}-1 \mathrm{~S}$, having a NIR nebula indicated by the extended circumstellar structures in the LP map, shows no extensive regions with significant $\mathrm{CP}$, and has very low $\mathrm{CP}$ measured through aperture polarimetry. The aperture polarimetry of several hundred point-like sources showed low $\mathrm{CP}$, indicating that low- or medium-mass young stars (i.e., sun-like stars) themselves do not show significant CP. If our solar system formed in a massive star-forming region (not in a low mass star-forming region) and was irradiated by asymmetric $\mathrm{CP}$, then EEs could have been produced in the parent bodies of the meteorites delivering an initial chiral bias of amino acids (or precursor) onto the early Earth.

Acknowledgements We thank the anonymous referee for a helpful review. We acknowledge discussions with T. Nagata, T. Nagayama, and S. Sato. We thank F. Palla for providing us with the table of the stellar model of Testi et al. (1998). T.F. was supported by Research Fellowships of the Japan Society for the Promotion of Science (JSPS) for Young Scientists. This work was partially supported by KAKENHI 183219. M.T. is supported by Grants-in-Aid from the Ministry of Education, Culture, Sports, Science and Technology (MEXT) of Japan (16077101, 16077204), and that from the JSPS (19204018). D.C.B.W. acknowledges support from the NASA Exobiology Program (grant NNX07AK38G) and the NASA Astrobiology Institute. IRAF is distributed by the National Optical Astronomy Observatories, which are operated by the Association of Universities for Research in Astronomy, Inc., under cooperative agreement with the National Science Foundation. 
Open Access This article is distributed under the terms of the Creative Commons Attribution Noncommercial License which permits any noncommercial use, distribution, and reproduction in any medium, provided the original author(s) and source are credited.

\section{References}

Bailey J, Chrysostomou A, Hough JH, Gledhill TM, McCall A, Clark S, Menard F, Tamura M (1998) Circular polarization in star-formation regions: implications for biomolecular homochirality. Science 281:672-674

Bailey J (2001) Astronomical sources of circularly polarized light and the origin of homochirality. Orig Life Evol Biosph 31:167-183

Barron LD (2008) Chirality and life. Space Sci Rev 135:187-201

Bernstein MP, Dworkin JP, Sandford SA, Cooper GW, Allamandola LJ (2002) Racemic amino acids from the ultraviolet photolysis of interstellar ice analogues. Nature 416:401-403

Beuther H, Zhang Q, Greenhill LJ, Reid MJ, Wilner D, Keto E, Marrone D, Ho PTP, Moran JM, RaoR SH, Liu SY (2004) Subarcsecond submillimeter continuum observations of Orion KL. Astrophys J 616:31-34

Bonner WA (1991) The origin and amplification of biomolecular chirality. Orig Life Evol Biosph 21:59-111

Bonner WA (1995) Chirality and life. Orig Life Evol Biosph 25:175-190

Bonner WA, Bean BD (2000) Asymmetric photolysis with elliptically polarized light. Orig Life Evol Biosph 30:513-517

Botta O, Bada JL (2002) Extraterrestrial organic compounds in meteorites. Surv Geophys 23:411-467

Buschermöhle M, Whittet DCB, Chrysostomou A, Hough JH, Lucas PW, Adamson AJ, Whitney BA, Wolff MJ (2005) An extended search for circularly polarized infrared radiation from the OMC-1 region of Orion. Astrophys J 624:821-826

Chrysostomou A, Ménard F, Gledhill TM, Clark S, Hough JH, McCall A, Tamura M (1997) Polarimetry of young stellar objects- II. Circular polarization of GSS 30. Mon Not R Astron Soc 285:750-758

Chrysostomou A, Gledhill TM, Menard F, Hough JH, Tamura M, Bailey J (2000) Polarimetry of young stellar objects- III. Circular polarimetry of OMC-1. Mon Not R Astron Soc 312:103-115

Chrysostomou A, Lucas PW, Hough JH (2007) Circular polarimetry reveals helical magnetic fields in the young stellar object HH135-136. Nature 450:71-73

Clark S, McCall A, Chrysostomou A, Gledhill T, Yates J, Hough J (2000) Polarization models of young stellar objects- II. Linear and circular polarimetry of R Coronae Australis. Mon Not R Astron Soc 319:337-349

Clayton GC, Whitney BA, Wolff MJ, Smith P, Gordon KD (2005) Circular polarization mapping of protostellar environments: searching for aligned grains. In: Adamson A et al (ed) Astronomical polarimetry: current status and future directions. ASP, San Francisco, 2005, ASP Conf. Ser. 343:122-127

Cohen JG, Persson SE, Elias JH, Frogel JA (1981) Bolometric luminosities and infrared properties of carbon stars in the Magellanic Clouds and the Galaxy. Astrophys J 249:481-503

Córdova A, Engqvist M, Ibrahem I, Casas J, Sunde'n H (2005) Plausible origins of homochirality in the amino acid catalyzed neogenesis of carbohydrates. Chem Commun 2047-2049

Córdova A, Zou W, Dziedzic P, Ibrahem I, Reyes E, Xu Y (2006) Direct asymmetric intermolecular Aldol reactions catalyzed by Amino Acids and small peptides. Chem Eur J 12:5383-5397

Cronin JR, Pizzarello S (1997) Enantiomeric excesses in meteoritic amino acids. Science 275:951-955

Fischer O, Henning T, Yorke HW (1996) Simulation of polarization maps. II. The circumstellar environment of pre-main sequence objects. Astron Astrophys 308:863-885

Fukue T, Tamura M, Kandori R, Kusakabe N, Hough JH, Lucas PW, Bailey J, Whittet DCB, Nakajima Y, Hashimoto J, Nagata T (2009) Near-infrared circular polarimetry and correlation diagrams in the Orion Becklin-Neugebauer/Kleinman-Low region: contribution of dichroic extinction. Astrophys J 692:88-91

Fürész G, Hartmann LW, Megeath ST, Szentgyorgyi AH, Hamden ET (2008) Kinematic structure of the Orion nebula cluster and its surroundings. Astrophys J 676:1109-1122

Genzel R, Stutzki J (1989) The Orion molecular cloud and star-forming region. Annu Rev Astron Astrophys 27:41-85

Getman KV, Feigelson ED, Grosso N, McCaughrean MJ, Micela G, Broos P, Garmire G, Townsley L (2005) Membership of the Orion nebula population from the Chandra Orion ultradeep project. Astrophys J Suppl Ser 160:353-378

Gezari DY (1992) Mid-infrared imaging of Orion BN/KL- Astrometry of IRc2 and the SiO maser. Astrophys J 396:43-47 
Glavin DP, Dworkin JP (2009) Enrichment of the amino acid l-isovaline by aqueous alteration on CI and CM meteorite parent bodies. Proc Natl Acad Sci USA 106:5487-5492

Gledhill TM, Chrysostomou A, Hough JH (1996) Linear and circular imaging polarimetry of the Chamaeleon infrared nebula. Mon Not R Astron Soc 282:1418-1436

Gledhill TM, McCall A (2000) Circular polarization by scattering from spheroidal dust grains. Mon Not R Astron Soc 314:123-137

Griesbeck AG, Meierhenrich UJ (2002) Asymmetric photochemistry and photochirogenesis. Angew Chem Int Ed 41:3147-3154

Hester JJ, Desch SJ (2005) Understanding our origins: star formation in HII region environments. In: Krot AN et al (ed) Chondrites and the protoplanetary disk, ASP, San Francisco, 2005, 341:107-130

Hester JJ, Desch SJ, Healy KR, Leshin LA (2004) The cradle of the solar system. Science 304:1116-1117

Hillenbrand LA (1997) On the stellar population and star-forming history of the Orion nebula cluster. Astron J 113:1733-1768

Hudson RL, Moore MH, Dworkin JP, Martin MP, Pozun ZD (2008) Amino Acids from ion-irradiated NitrileContaining ices. Astrobiology 8:771-779

Jiang Z, Tamura M, Fukagawa M, Hough J, Lucas P, Suto H, Ishii M, Yang J (2005) A circumstellar disk associated with a massive protostellar object. Nature 437:112-115

Jones BF, Walker MF (1988) Proper motions and variabilities of stars near the Orion nebula. Astron J 95:1755-1782

Kandori R, Kusakabe N, Tamura M, Nakajima Y, Nagayama T, Nagashima C, Hashimoto J, Hough J, Sato S, Nagata T, Ishihara A, Lucas P, Fukagawa M (2006) SIRPOL: a JHKs-simultaneous imaging polarimeter for the IRSF 1.4-m telescope. Proc SPIE 6269:159

Klussmann M, Iwamura H, Mathew SP, Wells DH, Pandya U, Armstrong A, Blackmond DG (2006) Thermodynamic control of asymmetric amplification in amino acid catalysis. Nature 441:621-623

Kusakabe N, Tamura M, Kandori R, Hashimoto J, Nakajima Y, Nagata T, Nagayama T, Hough J, Lucas P (2008) Near-infrared imaging polarimetry of M42: aperture polarimetry of point-like sources. Astron J 136:621-630

Lucas PW, Roche PF, Allard F, Hauschildt PH (2001) Infrared spectroscopy of substellar objects in Orion. Mon Not R Astron Soc 326:695-721

Lucas PW, Fukagawa M, Tamura M, Beckford AF, Itoh Y, Murakawa K, Suto H, Hayashi SS, Oasa Y, Naoi T, Doi Y, Ebizuka N, Kaifu N (2004) High-resolution imaging polarimetry of HL Tau and magnetic field structure. Mon Not R Astron Soc 352:1347-1364

Lucas PW, Hough JH, Bailey J, Chrysostomou A, Gledhill TM, McCall A (2005) UV circular polarisation in star formation regions: the origin of homochirality? Orig Life Evol Biosph 35:29-60

Meierhenrich UJ, Thiemann WH-P (2004) Photochemical concepts on the origin of biomolecular asymmetry. $12134: 111-121$

Meierhenrich UJ, Muñoz Caro GM, Schutte WA, Thiemann WH-P, Barbier B, Brack A (2005a) Precursors of biological cofactors from ultraviolet irradiation of circumstellar/interstellar ice analogs. Chem Eur J 11:4895-4900

Meierhenrich UJ, Nahon L, Alcaraz C, Bredehöft JH, Hoffmann SV, Barbier B, Brack A (2005b) Asymmetric vacuum UV photolysis of the Amino Acid Leucine in the Solid State. Angew Chem Int Ed 44:5630-5634

Ménard F, Chrysostomou A, Gledhill T, Hough JH, Bailey J (2000) High circular polarization in the star forming region NGC 6334: Implications. In: Lemarchand G, Meech K (ed) Bioastronomy 99: a new era in the search for Life in the Universe, San Francisco, ASP Conf. 213:355-358

Minchin NR, Hough JH, McCall A, McCaughrean BMG, MJ AC, Bailey JA, Axon DJ, Sato S (1991) Nearinfrared imaging polarimetry of bipolar nebulae. I - The BN-KL region of OMC-1. Mon Not R Astron Soc 248:715-729

Mostefaoui S, Lugmair GW, Hoppe P (2005) ${ }^{60} \mathrm{Fe}$ : a heat source for planetary differentiation from a nearby supernova explosion. Astrophys J 625:271-277

Muñoz-Caro GM, Meierhenrich UJ, Schutte WA, Barbier B, Arcones Segovia A, Rosenbauer H, Thiemann WHP, Brack A, Greenberg JM (2002) Amino acids from ultraviolet irradiation of interstellar ice analogues. Nature 416:403-406

Nagayama T, Nagashima C, Nakajima Y, Nagata T, Sato S, Nakaya H, Yamamuro T, Sugitani K, Tamura M (2003) SIRUS: a near infrared simultaneous three-band camera. Proc SPIE 4841:459-464

Nuevo M, Meierhenrich UJ, Muñoz Caro GM, Dartois E, D’Hendecourt L, Deboffle D, Auger G, Blanot D, Bredehöft J-H, Nahon L (2006) The effects of circularly polarized light on amino acid enantiomers produced by the UV irradiation of interstellar ice analogs. Astron Astrophys 457:741-751

Nuevo M, Auger G, Blanot D, d'Hendecourt L (2008) A detailed study of the amino acids produced from the vacuum UV irradiation of interstellar ice analogs. Orig Life Evol Biosph 38:37-56

O'dell CR (2001) The Orion nebula and its associated population. Annu Rev Astron Astrophys 39:99-136 
Ouellette N, Desch SJ, Hester JJ (2007) Interaction of supernova Ejecta with nearby protoplanetary disks. Astrophys J 662:1268-1281

Pizzarello S, Cronin JR (2000) Non-racemic amino acids in the Murray and Murchison meteorites. Geochim Cosmochim Acta 64:329-338

Pizzarello S, Zolensky M, Turk KA (2003) Nonracemic isovaline in the Murchison meteorite: chiral distribution and mineral association. Geochim Cosmochim Acta 67:1589-1595

Pizzarello S, Weber AL (2004) Prebiotic amino acids as asymmetric catalysts. Science 303:1151

Pizzarello S, Huang Y, Alexandre MR (2008) Molecular asymmetry in extraterrestrial chemistry: insights from a pristine meteorite. PNAS 105:3700-3704. doi:10.1073/pnas.0709909105

Rubenstein E, Bonner WA, Noyes HP, Brown GS (1983) Supernovae and life. Nature 306:118

Sephton MA (2002) Organic compounds in carbonaceous meteorites. Nat Prod Rep 19:292-311

Shibata T, Yamamoto J, Matsumoto N, Yonekubo S, Osanai S, Soai K (1998) Amplification of a slight enantiomeric imbalance in molecules based on asymmetric autocatalysis: the first correlation between high enantiomeric enrichment in a Chiral molecule and circularly polarized light. J Am Chem Soc 120:12157-12158

Simpson JP, Colgan SWJ, Erickson EF, Burton MG, Schultz ASB (2006) Hubble space telescope NICMOS polarization measurements of OMC-1. Astrophys J 642:339-353

Soai K, Shibata T, Morioka H, Choji K (1995) Asymmetric autocatalysis and amplification of enantiomeric excess of a chiral molecule. Nature 378:767-768

Soai K, Kawasaki T (2006) Discovery of asymmetric autocatalysis with amplification of chirality and its implications in chiral homogeneity of biomolecules. Chirality 18:469-478

Tachibana S, Huss GR, Kita NT, Shimoda G, Morishita Y (2006) ${ }^{60}$ Fe in Chondrites: debris from a nearby supernova in the early solar system? Astrophys J 639:L87-L90

Takano Y, Takahashi J, Kaneko T, Marumo K, Kobayashi K (2007) Asymmetric synthesis of amino acid precursors in interstellar complex organics by circularly polarized light. Earth Planet Sci Lett 254:106-114

Tamura M, Fukagawa M, Murakawa K, Suto H, Itoh Y, Doi Y (2003) Near-infrared polarimeter for the Subarau telescope. SPIE 4843:190-195

Tamura M, Kandori R, Kusakabe N, Nakajima Y, Hashimoto J, Nagashima C, Nagata T, Nagayama T, Kimura H, Yamamoto T, Hough JH, Lucas P, Chrysostomou A, Bailey J (2006) Near-infrared polarization images of the Orion nebula. Astrophys J 649:L29-L32

Testi L, Palla F, Natta A (1998) A search for clustering around Herbig Ae/Be stars. II. Atlas of the observed sources. Astron Astrophys 133:81-121

Weber AL (2001) The sugar model: catalysis by amines and amino acid products. Orig Life Evol Biosph 31:71-86

Whitney BA, Wolff MJ (2002) Scattering and absorption by aligned grains in circumstellar environments. Astrophys J 574:205-231

Wolf S, Voshchinnikov NV, Henning T (2002) Multiple scattering of polarized radiation by non-spherical grains: first results. Astron Astrophys 385:365-376 